\title{
MRI Natural History of the Leukodystrophy Vanishing White Matter
}

\author{
Menno D. Stellingwerff, MD • Murtadha L. Al-Saady, MD • Tim van de Brug, PhD • Frederik Barkhof, MD, PhD • \\ Petra J. W. Pouwels, PhD • Marjo S. van der Knaap, MD, PhD
}

\begin{abstract}
From the Department of Child Neurology, Emma Children's Hospital, Amsterdam University Medical Centers, Vrije Universiteit and Amsterdam Neuroscience, De Boelelaan 1117, Amsterdam 1081 HV, the Netherlands (M.D.S., M.L.A., M.S.v.d.K.); Department of Epidemiology and Data Science, Amsterdam University Medical Centers, Amsterdam, the Netherlands (T.v.d.B.); Department of Radiology and Nuclear Medicine, Amsterdam University Medical Center, Vrije Universiteit and Amsterdam Neuroscience, Amsterdam, the Netherlands (F.B., P.J.W.P.); Institutes of Neurology and Health Care Engineering, University College London, London, England (F.B.); and Department of Functional Genomics, Center for Neurogenomics and Cognitive Research, VU University, Amsterdam, the Netherlands (M.S.v.d.K.). Received January 19, 2021; revision requested March 16; revision received April 6; accepted April 19. Address correspondence to M.S.v.d.K. (e-mail: ms.vanderknaap@amsterdamumc.n1).

F.B. supported by the National Institute for Health Research Biomedical Research Centre at University College London Hospital.
\end{abstract}

Conflicts of interest are listed at the end of this article.

Radiology 2021; 000:1-9 • https://doi.org/10.1148/radiol.2021210110 • Content codes: NR MR

Background: In vanishing white matter (VWM), a form of leukodystrophy, earlier onset is associated with faster clinical progression. MRI typically shows rarefaction and cystic destruction of the cerebral white matter. Information on the evolution of VWM according to age at onset is lacking.

Purpose: To determine whether nature and progression of cerebral white matter abnormalities in VWM differ according to age at onset.

\begin{abstract}
Materials and Methods: Patients with genetically confirmed VWM were stratified into six groups according to age at onset: younger than 1 year, 1 year to younger than 2 years, 2 years to younger than 4 years, 4 years to younger than 8 years, 8 years to younger than 18 years, and 18 years or older. With institutional review board approval, all available MRI scans obtained between 1985 and 2019 were retrospectively analyzed with three methods: (a) ratio of the width of the lateral ventricles over the skull (ventricle-to-skull ratio [VSR]) was measured to estimate brain atrophy; (b) cerebral white matter was visually scored as percentage normal, hyperintense, rarefied, or cystic on fluid-attenuated inversion recovery (FLAIR) images and converted into a white matter decay score; and (c) the intracranial volume was segmented into normal-appearing white and gray matter, abnormal but structurally present (FLAIRhyperintense) and rarefied or cystic (FLAIR-hypointense) white matter, and ventricular and extracerebral cerebrospinal fluid (CSF). Multilevel regression analyses with patient as a clustering variable were performed to account for the nested data structure.
\end{abstract}

Results: A total of 461 examinations in 270 patients (median age, 7 years [interquartile range, 3-18 years]; 144 female patients) were evaluated; 112 patients had undergone serial imaging. Patients with later onset had higher VSR $[\mathrm{F}(5)=8.42 ; P<.001]$ and CSF volume $[\mathrm{F}(5)=21.7 ; P<.001]$ and lower white matter decay score $[\mathrm{F}(5)=4.68 ; P<.001]$ and rarefied or cystic white matter volume $[\mathrm{F}(5)=13.3 ; P<.001]$. Rate of progression of white matter decay scores $[\mathrm{b}=-1.6, \mathrm{t}(109)=-3.9 ; P<.001]$ and VSRs $[\mathrm{b}=-0.05, \mathrm{t}(109)=-3.7 ; P<.001]$ were lower with later onset.

Conclusion: A radiologic spectrum based on age at onset exists in vanishing white matter. The earlier the onset, the faster and more cystic the white matter decay, whereas with later onset, white matter atrophy and gliosis predominate.

(C)RSNA, 2021

In vanishing white matter (VWM), a form of leukodystrophy, the cerebral white matter is the only or the most prominently affected tissue (1). The MRI feature defining VWM is progressive rarefaction and cystic decay of the cerebral white matter, leading to a white matter signal close to or the same as that of cerebrospinal fluid (CSF) on proton density and fluid-attenuated inversion recovery (FLAIR) images (1). The rarefied and cystic white matter typically contains radiating stripes, suggesting relatively preserved perivascular tissue strands (1). The white matter involvement is extensive or diffuse (1); only the directly subcortical white matter may be spared (2). Neuropathologic evaluation confirms the interpretation of the MRI findings (3).

VWM is caused by biallelic sequence variations in any of the EIF2B1-5 genes, which encode the five subunits of the eukaryotic translation initiation factor eIF2B $(4,5)$. The onset of VWM is mainly in early childhood but ranges from the antenatal period to senescence (6). For onset before age
4 years, earlier manifestation is associated with faster clinical disease progression. Patients with onset after age 4 years tend to have a milder disease course, irrespective of the exact age at onset (6).

Currently, there is no therapy for VWM, but various drugs are in clinical development and will be assessed in trials (7-9). Although clinical parameters provide primary outcome measures, MRI parameters may serve as secondary or surrogate outcome measures. For the past 3 decades, MRI has played an important role in the diagnosis of VWM (1). The time course of the cerebral white matter decay has, however, not been investigated, and systematic information on MRI evolution for different ages of onset is lacking. For MRI to play a role in therapeutic trials, this basic information is needed. We therefore studied the progression of cerebral white matter abnormalities at MRI in a large group of patients with genetically confirmed VWM, representing all ages of onset. 


\section{Abbreviations}

$\mathrm{CSF}=$ cerebrospinal fluid, FLAIR $=$ fluid-attenuated inversion recovery, IQR = interquartile range, VSR = ventricle-to-skull ratio, $\mathrm{VWM}=$ vanishing white matter

\section{Summary}

Pathologic features and progression of cerebral white matter decay markedly vary in vanishing white matter; earlier onset is associated with faster and more cystic decay, whereas atrophy and gliosis predominate with later onset.

\section{Key Results}

- In vanishing white matter, a form of leukodystrophy, earlier onset correlates with faster progression $[\mathrm{b}=-1.6, \mathrm{t}(109)=-3.9 ; P<.001]$ and more rarefying and cystic cerebral white matter decay $[\mathrm{F}(5)=13.3$; $P<.001]$, whereas later onset correlates with more atrophy and gliosis of the cerebral white matter $[\mathrm{F}(5)=21.7 ; P<.001]$.

- The differences between age-at-onset groups likely reflect the severity of astrocytic dysfunction.

\section{Materials and Methods}

\section{Patients with VWM and MRI Examinations}

The local ethics committee approved this retrospective study. Informed consent was obtained from patients and/or their parents or guardians. All MRI scans of patients with VWM in the Amsterdam leukodystrophy database were considered. The only inclusion criteria were genetic confirmation of VWM and availability of at least one MRI scan (279 patients, 479 MRI scans). The only exclusion criterion was an additional neurologic disease (nine patients, $18 \mathrm{MRI}$ scans). Thus, the total numbers of patients and MRI examinations in the study were 270 and 461, respectively.

MRI examinations came from all over the world, were performed between 1985 and 2019, and were obtained by us in the context of second opinions, diagnostic testing, and the VWM clinical natural history study (6). MRI examinations had been performed with use of numerous different MRI machines from different vendors and numerous different pulse sequences. Radiologic films were digitized. Consequently, not all methods could be applied to all examinations, and in the absence of homogeneous data sets, no analysis of quantitative MRI parameters could be performed.

Age at onset is the only strong predictor of clinical course and outcome $(6,10)$. Sequence variant information does not add predictive information (11). Patients were therefore stratified into six groups according to age at onset of VWM, as follows: younger than 1 year (group 1), 1 year to younger than 2 years (group 2), 2 years to younger than 4 years (group 3), 4 years to younger than 8 years (group 4), 8 years to younger than 18 years (group 5), and 18 years or older (group 6), as previously described (6). Clinical details of 236 patients were reported previously (6), and MRI examinations of 20 patients were described previously (12-14). Clinical investigations and MRI examinations were not performed at the same time and could not be correlated.

\section{Ventricle-to-Skull Ratio}

For all MRI examinations, the ventricle-to-skull ratio (VSR) was determined on axial images. The VSR was defined as the sum of the maximum transverse diameters of the lateral ventricles at the level of the frontal horns, occipital horns, and cella media divided by the maximum transverse internal skull diameter (Fig 1) (15). These measures were performed with a Centricity RA 600 workstation (version 6.1; GE Medical Systems).

\section{White Matter Decay Score}

For originally digitally available examinations with FLAIR and T1-weighted sequences, we calculated a white matter decay score. Because of the moderate quality of digitized films, they were not included in this analysis. White matter was visually divided into the following categories: (a) normal-appearing, (b) FLAIR-hyperintense, (c) rarefied, and (d) cystic. Normalappearing white matter was defined as FLAIR-hypointense and T1-hyperintense. FLAIR-hyperintense white matter was defined as being hyperintense compared with the cortex. Rarefied white matter was defined at FLAIR as having a higher signal intensity than CSF but a lower signal intensity than normal white matter. Cystic white matter was defined as being isointense to CSF at FLAIR. The estimated percentages of all areas compared with the total white matter volume were weighted ([percentage normal-appearing white matter $\times 0$ ] + [percentage FLAIRhyperintense white matter $\times 10]+$ [percentage rarefied white matter $\times 20]+$ [percentage cystic white matter $\times 30])$, yielding a white matter decay score ranging from 0 (100\% normal-appearing white matter) to 30 (100\% cystic white matter). Because normal white matter is FLAIR-hyperintense in patients younger than 1 year, we rated FLAIR-hyperintense white matter in these patients as normal appearing.

\section{Segmentation}

Because MRI examinations were performed for clinical purposes and no single segmentation program reliably segmented all images, various segmentation programs were combined.

Preprocessing.-T1-weighted and FLAIR images were used for segmentation. Scans with coverage less than the full supratentorial volume were discarded. Images were interpolated to $1-\mathrm{mm}$ isometric voxels by using the freely available Functional Magnetic Resonance Imaging of the Brain's Linear Image Registration Tool, or FLIRT (version 5.0). FLAIR images were registered to T1-weighted images by using the open-source software nicMSlesions (version 0.2) (16). The open-source software Statistical Parametric Mapping, or SPM12, segment (version 12.7219; Wellcome Centre for Human Neuroimaging, University College London Queen Square Institute of Neurology) was applied to remove the skull and extracranial tissue image segments.

Segmentation.-Intracranial volume was segmented into five tissue classes: (a) normal-appearing tissue, including both cortex and white matter; (b) FLAIR-hyperintense white matter; (c) rarefied or cystic white matter; (d) ventricular CSF; and (e) extracerebral CSF. FLAIR-hyperintense voxels were segmented by using nicMSlesions (16). The Functional Magnetic Resonance Imaging of the Brain's Automated Segmentation Tool, or FAST, with two-class segmentation was used to segment CSF-like voxels from tissue-like voxels on T1-weighted images. 


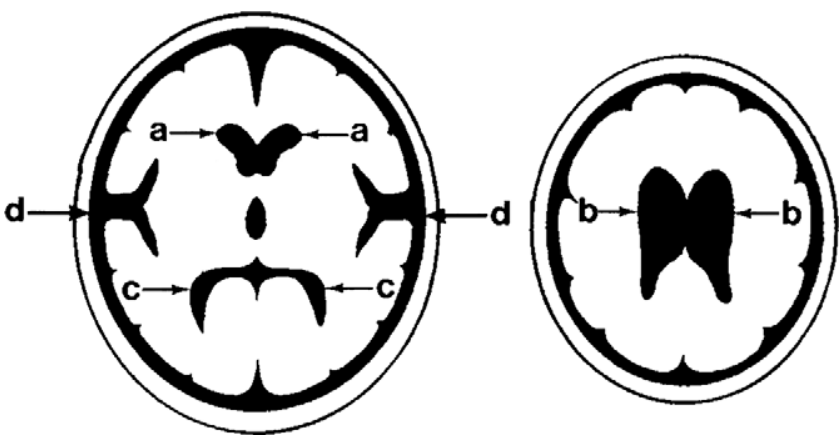

Figure 1: Diagram depicts the ventricle-to-skull ratio (VSR), which is defined as the sum of the maximum transverse diameters of the lateral ventricles at the level of the frontal horns (a), occipital horns (c), and cella media (b) divided by the maximum transverse internal skull diameter (d). (Adapted, with permission, from reference 15.)

CSF-like voxels were separated manually into rarefied or cystic white matter, ventricles, and extracerebral CSF. The remaining voxels were classified as normal-appearing tissue.

Postprocessing. - After each segmentation step, manual corrections were made if necessary. Only supratentorial voxels were included in the analyses by converting a supratentorial mask from Montreal Neurological Institute space to subject space by using the inverse deformation field acquired with the Statistical Parametric Mapping segment. Percentages, determined by the tissue classes' volume divided by the total supratentorial intracranial volume, were used for analyses.

\section{Comparing Methods}

The VSR was compared with the segmented ventricular volume as percentage of the total supratentorial intracranial volume. Segmentation and visual scoring were compared by assessing the ratio of FLAIR-hyperintense white matter volume over abnormal white matter volume (ie, the sum of FLAIRhyperintense and cystic or rarefied white matter) as determined with both methods. Total white matter could not be used as the denominator because segmentation did not differentiate between normal white matter and the cortex.

\section{Statistical Analysis}

All analyses were performed by one author (M.D.S., with 2.5 years of experience in MRI analysis) with use of the commercially available RStudio software (version 1.1.463; RStudio Team) under supervision of another author (T.v.d.B., with 15 years of experience in statistics). $P<.05$ was considered indicative of a statistically significant difference. Nonparametric data were displayed as medians with interquartile ranges (IQRs). Several patients underwent multiple MRI examinations, and the data were therefore clustered. Because the assumption of independent observations was not met, multilevel analyses were performed with patient as a clustering variable, with the ordinal groups as a predictor for group analysis and the continuous log-transformed age at onset as a predictor for regression analysis. $F$ values are reported for size of group differences, $b$ values for the slope of the regression predictors, and the $t$ statistic as indicator of precision of the regression predic- tor. To analyze progression in patients with multiple examinations, multiple regression modeling was performed on the difference between the first and last examination, with age at onset and time interval as independent variables. The Spearman correlation coefficient was used to compare methods, as described earlier.

Observers were trained in MRI interpretation by one author (M.S.v.d.K., with 34 years of experience in MRI of leukodystrophies) and in MRI segmentation by another author (P.J.W.P., with 25 years of experience in MRI physics). One observer (M.D.S.) performed all measurements used in the analyses. Fifty randomly selected examinations were reanalyzed by the same observer 6 months later and a second observer (M.L.A., with 5 years of experience in MRI analysis) to determine intra- and interobserver reliability for the VSR and white matter decay score. Observers were blinded to age at onset. The intraclass correlation coefficient was considered fair to good when greater than 0.4 and excellent when greater than 0.75 (17).

\section{Results}

\section{Patients and MRI Examinations}

In total, $461 \mathrm{MRI}$ examinations in 270 patients (median age, 7 years [IQR, 3-18 years]; 144 female and 126 male patients) were available (Table). Of these, 120 were digitized films. A total of 191 follow-up examinations performed in 112 patients were available. Examples of MRI scans for different ages at onset are shown in Figure 2. In total, 323 examinations in 206 patients were suitable for estimating the white matter decay score, and 149 examinations in 113 patients were suitable for segmentation; the other digitally available examinations either did not have sufficient brain coverage or suffered from motion artifacts.

\section{VSR}

The VSR was measured in all 461 MRI examinations (Fig 3). The lowest median VSR was present in group 3 (0.84 [IQR, $0.77-0.96])$ and the highest in group 6 (1.08 [IQR, 1.011.22]). Overall, the VSR was higher with older age at onset, except for group 1, in which the median VSR was higher than in group 2. The VSR differed significantly between the age-atonset groups $[\mathrm{F}(5)=8.42, P<.001]$, and its $\mathrm{U}$ shape can be described by the following quadratic regression equation:

$$
\begin{aligned}
\mathrm{VSR}= & 0.909-0.014 \times \ln (\text { age at onset })+0.021 \\
& \times[\ln (\text { age at onset })]^{2} .
\end{aligned}
$$

The difference in VSR ( $\Delta$ VSR) between the first and latest examination of patients can be described by the following regression equation:

$$
\begin{aligned}
\Delta \mathrm{VSR}= & 0.15-0.05 \times \ln (\text { age at onset })+0.03 \\
& \times \ln (\text { time interval }) .
\end{aligned}
$$

This indicates that the rate of progression is higher for patients with a younger age at onset $[\mathrm{b}=-0.05, \mathrm{t}(109)=-3.7$; $P<.001]$ and also after adjustment for the interval between the examinations $[\mathrm{b}=0.03, \mathrm{t}(109)=2.4 ; P=.02]$. The intraobserver reliability (intraclass correlation coefficient, 0.96; 95\% 


\begin{tabular}{|c|c|c|c|c|c|c|c|}
\hline \multirow[b]{2}{*}{ Variable } & \multirow[b]{2}{*}{ All Patients } & \multicolumn{6}{|c|}{ Age at Onset } \\
\hline & & $<1$ year & 1 to $<2$ years & 2 to $<4$ years & 4 to $<8$ years & 8 to $<18$ years & $\geq 18$ years \\
\hline No. of patients* & $270(100)$ & $34(13)$ & $45(17)$ & $73(27)$ & $51(19)$ & $29(11)$ & $38(14)$ \\
\hline $\begin{array}{c}\text { No. of female } \\
\text { patients* }\end{array}$ & $144(53)$ & $14(41)$ & $20(44)$ & $42(58)$ & $25(49)$ & $16(55)$ & $27(71)$ \\
\hline $\begin{array}{l}\text { No. of MRI } \\
\text { examinations* }\end{array}$ & $461(100)$ & $48(10)$ & $71(15)$ & $116(25)$ & $101(22)$ & $66(14)$ & $59(13)$ \\
\hline $\begin{array}{l}\text { Median age at } \\
\text { MRI (y) }\end{array}$ & $6.6(3.0-18.2)$ & $0.6(0.3-0.9)$ & $2.3(1.9-3.7)$ & $4.0(3.1-6.3)$ & $8.9(5.8-14.7)$ & $17.5(12.9-28.9)$ & $43.4(26.3-51.3)$ \\
\hline $\begin{array}{c}\text { Median disease } \\
\text { duration (y) }\end{array}$ & $1.7(0.5-7.1)$ & $0.2(0.1-0.3)$ & $1.1(0.3-2.6)$ & $1.2(0.7-3.5)$ & $3.6(0.9-8.6)$ & $4.5(1.0-16.2)$ & $6.1(2.6-15.0)$ \\
\hline $\begin{array}{c}\text { Median age at } \\
\text { onset }(y)\end{array}$ & $3.0(1.9-9.0)$ & $0.3(0.2-0.6)$ & $1.5(1.2-1.7)$ & $3.0(2.0-3.0)$ & $5.0(4.0-7.0)$ & $11.0(9.0-14.0)$ & $29.0(20.0-39.0)$ \\
\hline $\begin{array}{l}\text { No. of MRI } \\
\text { examinations } \\
\text { with VSR } \\
\text { determined }^{\dagger}\end{array}$ & $461(100)$ & $48(100)$ & $71(100)$ & $116(100)$ & $101(100)$ & $66(100)$ & $59(100)$ \\
\hline Median VSR & $0.93(0.83-1.07)$ & $0.93(0.86-1.03)$ & $0.86(0.80-0.92)$ & $0.84(0.77-0.96)$ & $0.99(0.85-1.08)$ & $0.98(0.91-1.09)$ & $1.08(1.01-1.22)$ \\
\hline $\begin{array}{l}\text { No. of } \\
\text { examinations } \\
\text { with white } \\
\text { matter decay } \\
\text { score calculated }^{\dagger}\end{array}$ & $323(70)$ & $32(67)$ & $48(68)$ & $81(70)$ & $68(67)$ & $48(73)$ & $46(78)$ \\
\hline $\begin{array}{l}\text { Median white } \\
\text { matter decay } \\
\text { score }\end{array}$ & $13.9(12.3-16.9)$ & $17.3(12.6-20.7)$ & $14.6(12.9-19.6)$ & $14.7(13.2-16.8)$ & $13.9(12.2-15.9)$ & $12.5(11.5-15.8)$ & $12.9(10.1-14.4)$ \\
\hline $\begin{array}{l}\text { No. of segmented } \\
\text { MRI } \\
\text { examinations }^{\dagger}\end{array}$ & $149(32)$ & $11(23)$ & $22(31)$ & $40(34)$ & $28(28)$ & $26(39)$ & $22(37)$ \\
\hline $\begin{array}{l}\text { Median rarefied } \\
(\%)\end{array}$ & $5.2(2.1-10.9)$ & $23.3(16.4-27.9)$ & $11.8(7.7-18.1)$ & $7.3(3.3-10.9)$ & $3.6(1.7-7.6)$ & $2.6(1.1-4.8)$ & $2.1(0.5-3.8)$ \\
\hline Median CSF (\%) & $25.5(19.4-31.4)$ & $17.7(15.7-21.6)$ & $17.9(15.3-21.3)$ & $21.6(17.5-25.9)$ & $31.0(25.1-32.9)$ & $30.0(26.0-32.5)$ & $33.9(29.5-40.8)$ \\
\hline
\end{tabular}

CI: $0.93,0.97)$ and interobserver reliability (intraclass correlation coefficient, 0.93; 95\% CI: 0.89, 0.95) were excellent.

\section{White Matter Decay Score}

The white matter decay score was visually determined for 323 MRI scans obtained in 206 patients (Fig 4); 117 of these were follow-up images obtained in 74 patients. Patients in group 1 had the highest median scores (17.3 [IQR, 12.6-20.7]), and those in group 5 had the lowest (12.5 [IQR, 11.5-15.8]). The white matter decay score significantly differed between age-atonset groups $[\mathrm{F}(5)=4.68 ; P<.001]$, and its negative linear$\log$ function can be described with the following regression equation:

white matter decay score $=16.2-1.2 \times \ln$ (age at onset) .

The difference in white matter decay score ( $\Delta$ WM decay) between the first and latest examination of patients can be described by the following regression equation:
$\Delta \mathrm{WM}$ decay $=6.4-1.6 \times \ln ($ age at onset $)+0.3$ $\times \ln$ (time interval).

This indicates that the rate of progression is higher for patients with a younger age at onset than for patients with older age at onset $[\mathrm{b}=-1.6, \mathrm{t}(109)=-3.9 ; P<.001]$; this was also seen after adjustment for the time interval between examinations $[\mathrm{b}=0.3, \mathrm{t}(109)=0.4 ; P=.42]$. Intraobserver reliability (intraclass correlation coefficient, 0.92; 95\% CI: 0.83 , 0.96 ) and interobserver reliability (intraclass correlation coefficient, 0.87 ; 95\% CI: 0.80, 0.92) were excellent.

\section{Segmentation}

In total, $149 \mathrm{MRI}$ examinations performed in 113 patients were segmented (Fig 5); 36 of these were follow-up examinations performed in 24 patients. The highest median percentage of rarefied or cystic white matter was present in group $1(23.3 \%$ [IQR, 16.4\%-27.9\%]) and the lowest in group $6(2.1 \%$ [IQR, $0.5 \%-3.8 \%])$. The percentage of rarefied or 

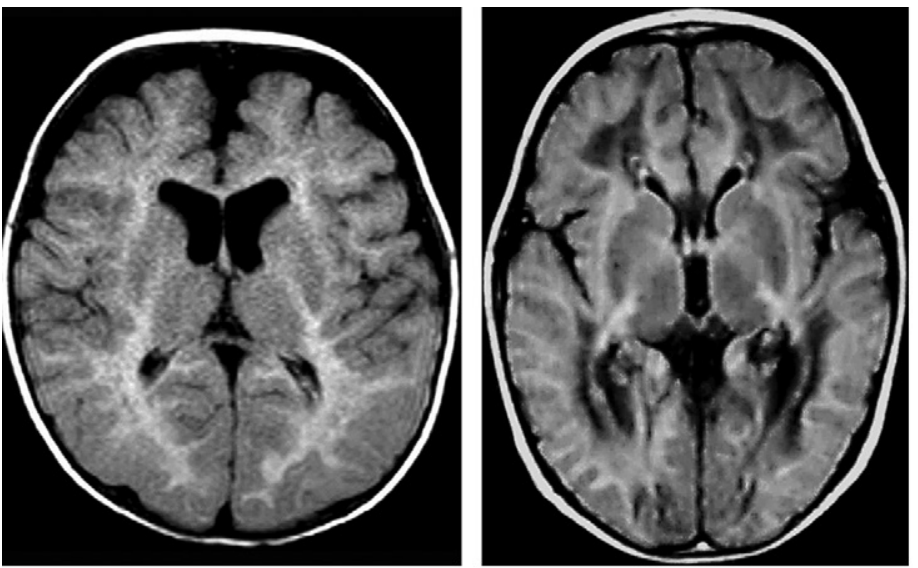

a.
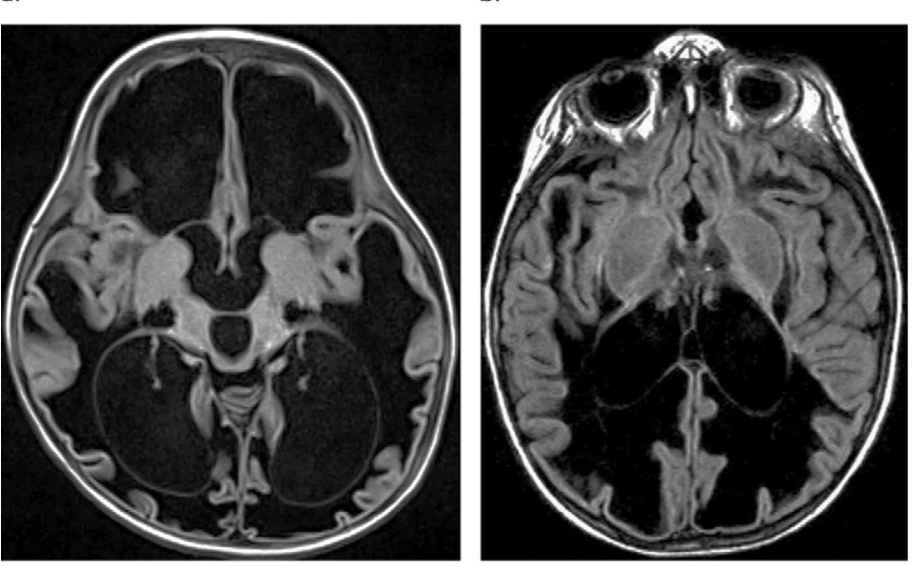
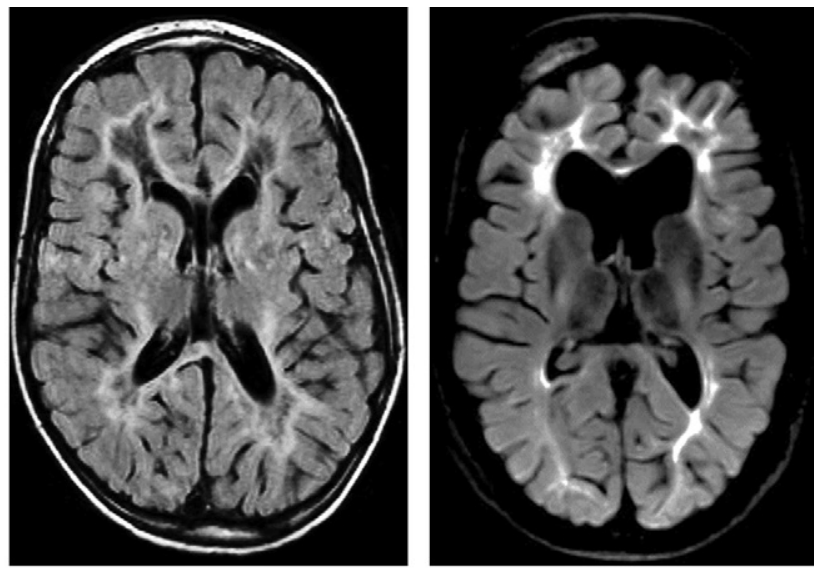

d.

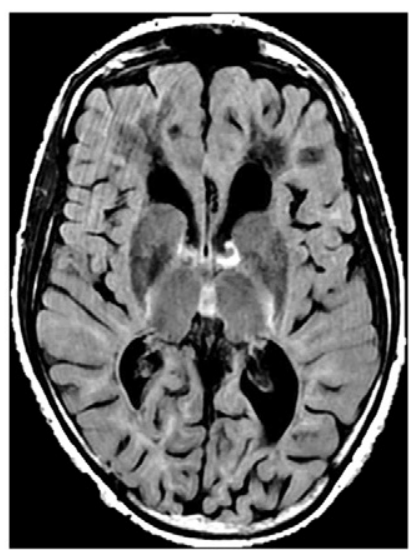

g.

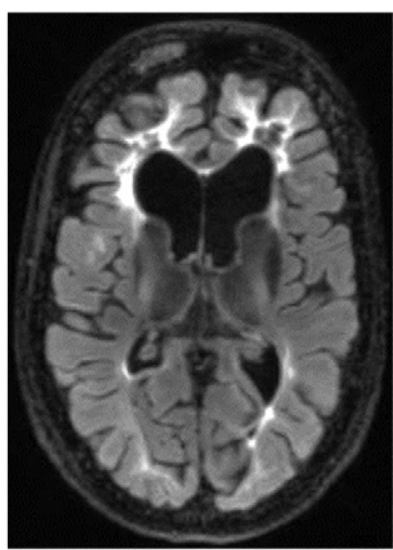

h.

Figure 2: Progression of white matter abnormalities at transverse fluid-attenuated inversion recovery (FLAIR) MRI examinations in patients with different ages at onset. (a, e) First (a) and last (e) MRI scans (at ages 7 months and 23 months) in a male patient with onset at 7 months. FLAIR hyperintensity of the internal capsule and corpus callosum and minimal rarefaction in the deep cerebral white matter are present on the first MRI scan (repetition time msec/echo time msec/inversion time msec, $6000 / 120 / 2000)$. Complete cystic degeneration of the cerebral white matter is visible on the last MRI scan (9774.8/109/2500). On the last MRI scan, the posterior part of the lateral ventricles is dilated because of white matter collapse; the anterior part of the ventricles has remained narrow, and the cystic white matter looks swollen. (b), f) First (b) and last (f) MRI scans (at ages 19 months and 30 months) in a male patient with onset at 14 months. The first MRI scan (8500/1 19/2500) shows diffuse white matter abnormalities with rarefaction in the deep white matter. On the last MRI scan (9000/105/2200), the cerebral white matter is fully replaced by fluid, and the cystic white matter looks swollen. $(\mathbf{c}, \mathbf{g})$ First $(\mathbf{c})(9000 / 105 / 2200)$ and last $(\mathbf{g})(9500 / 126.3 / 2250)$ MRI scans (at ages 8 years and 25 years) in a female patient with onset at 7 years. The deep white matter contains radiating stripes, reflecting better-preserved perivascular tissue strands within rarefied tissue. The last MRI scan shows a subtle increase in rarefied white matter and atrophy of the cerebral white matter. (d, h) First (d) and last (h) MRI examinations (at 48 years and 55 years) of a female patient with onset at age 46 years. The first MRI scan (8000/126.3/2347) shows mild rarefaction of the periventricular white matter, gliosis of the deep and subcortical white matter, and cerebral atrophy. The last MRI scan (8000/127.3/2344) displays no evident increase in rarefaction and a slight increase in cerebral atrophy.

cystic white matter significantly differed between age-at-onset groups $[\mathrm{F}(5)=13.3 ; P<.001]$, and its negative log-slope can be described by the following regression equation:

percentage rarefied or cystic

white matter $=13.53-3.66 \times \ln ($ age at onset $)$,

indicating less rarefied or cystic white matter with older age at onset. The lowest median CSF percentage was present in group $1(17.7 \%$ [IQR, 15.7\%-21.6\%]) and the highest in group 6 (33.9\% [IQR, 29.5\%-40.8\%]). The CSF percentage significantly differed between age-at-onset groups $[\mathrm{F}(5)=$ 21.7; $P<.001]$, and its positive log-slope can be described by the following regression equation:

percentage $\mathrm{CSF}=19.36+4.12 \times \ln ($ age at onset $)$, indicating more atrophy with an older age at onset. Because follow-up segmentation data were available in only $24 \mathrm{pa}-$ tients, multiple regression modeling was not performed.

\section{Comparing Methods}

The different methods were compared by determining their correlation (Fig 6). The VSR and the segmented ventricular volume as percentage of supratentorial intracranial volume were highly correlated $\left(r_{\mathrm{s}}=0.90 ; P<.001\right)$. The segmented and visually determined FLAIR-hyperintense volumes were also strongly correlated $\left(r_{\mathrm{s}}=0.86 ; P<.001\right)$. As the Bland-Altman plot suggests, the visually estimated volume tended to be higher than the segmented volume in the case of a low volume of FLAIRhyperintense white matter and vice versa. This can be explained by the fact that visual rating was done per 10 percentage points 

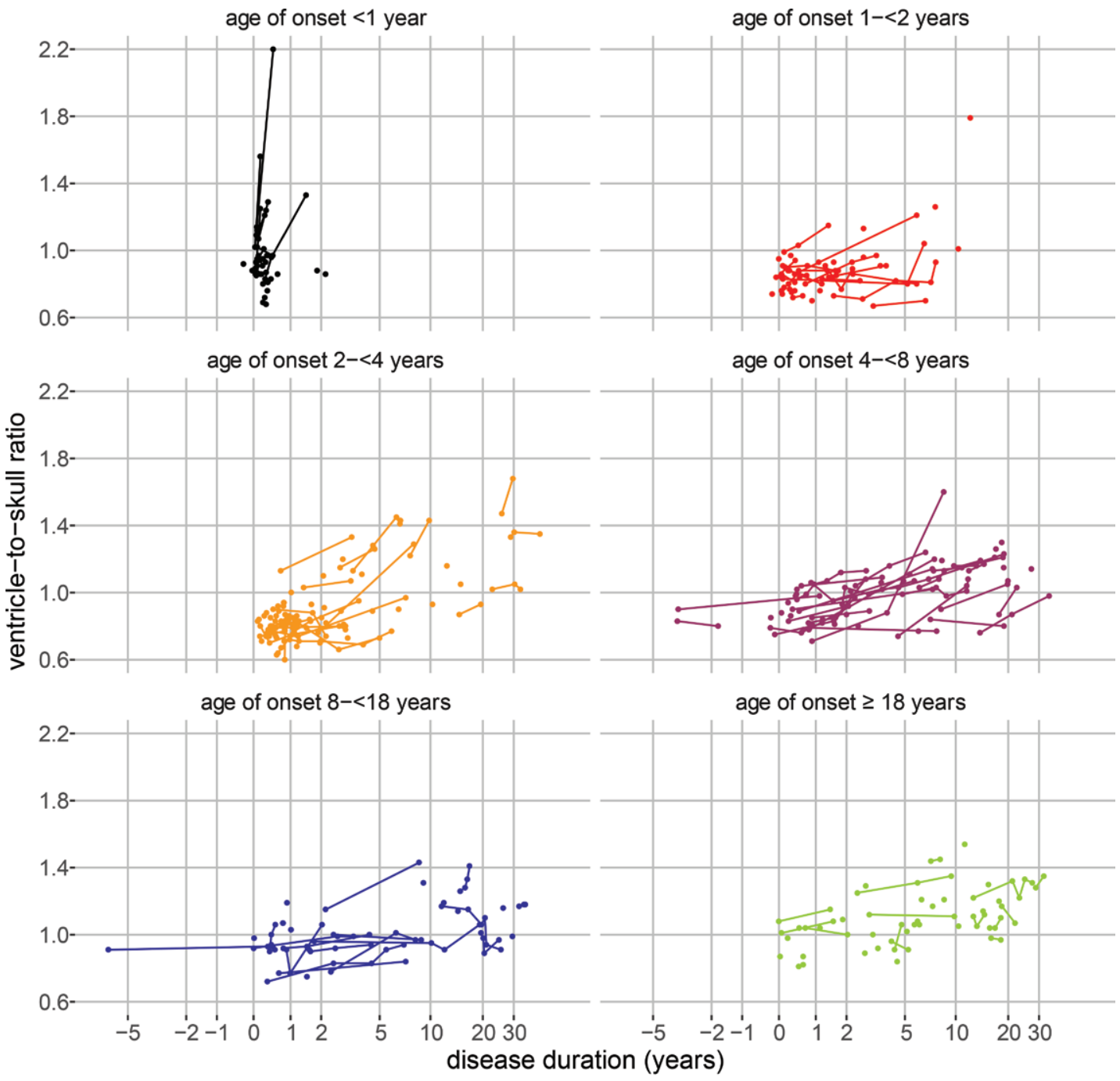

a.

$1.6-$

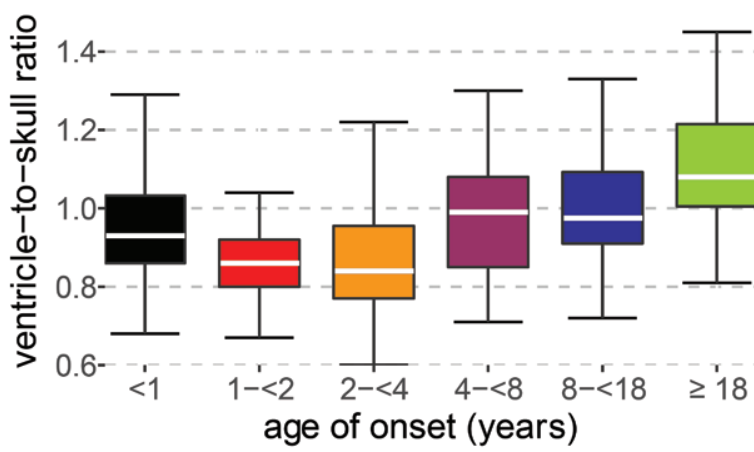

b.

(eg, $0 \%, 10 \%, 20 \%$ ). A lower percentage was rated higher, as it would otherwise be noted as absent. The mean difference \pm standard deviation between the methods was $1.3 \% \pm 13.9$.

\section{Discussion}

Although MRI plays a central role in the diagnosis and follow-up of vanishing white matter (VWM) by being able to help distinguish between different pathologic types of white matter-such
Figure 3: (a) Graphs show the ventricle-to-skull ratio (VSR) per age-at-onset group, plotted over the disease duration on a log scale to prevent cluttered data points close to disease onset. Negative values indicate presymptomatic examinations. Forty-eight examinations were included in group 1, 71 in group 2, 116 in group 3, 101 in group 4, 66 in group 5, and 59 in group 6. Points representing examinations of the same patients are connected. The slope of the change in VSR in the earlier age-at-onset groups is steeper. (b) Box plots show VSR according to age at onset, with medians (white bar), first and third quartiles (box), and the minimum and maximum values within 1.5 times the interquartile range (whiskers). The median VSR differs per age-at-onset group $(P<.01)$ and follows a skewed U shape.

as rarefaction, cystic degeneration, and gliosis-no comprehensive information is available on type and evolution of cerebral white matter decay at MRI. We performed a systematic analysis of 461 MRI examinations from 270 patients with VWM representing the entire clinical spectrum. Analyses were stratified by age at onset. Our study reveals an MRI phenotypic spectrum: With increasing age at onset, ventricle-to-skull ratio (VSR) and cerebrospinal fluid volume are higher, white matter decay 

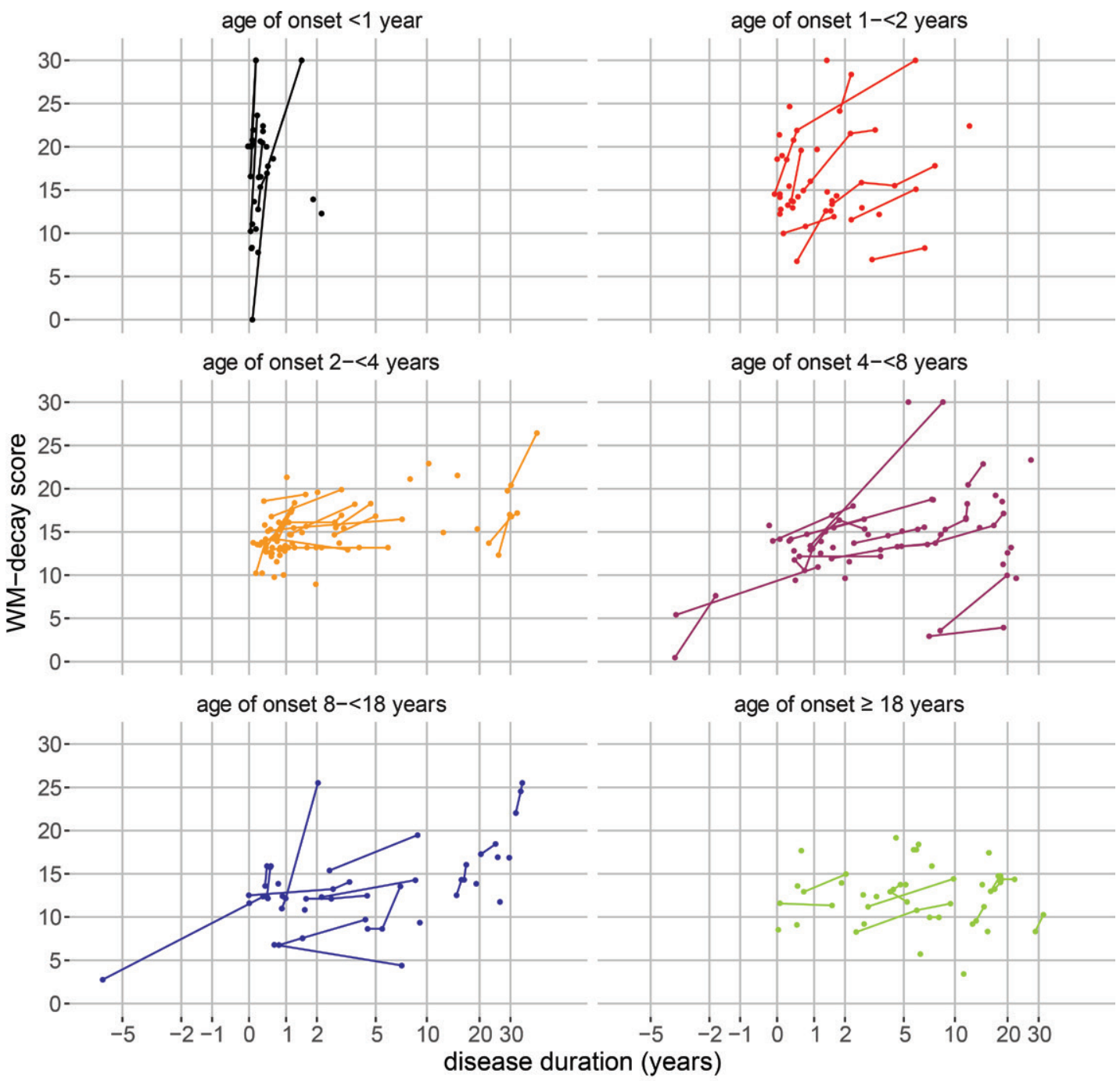

a.

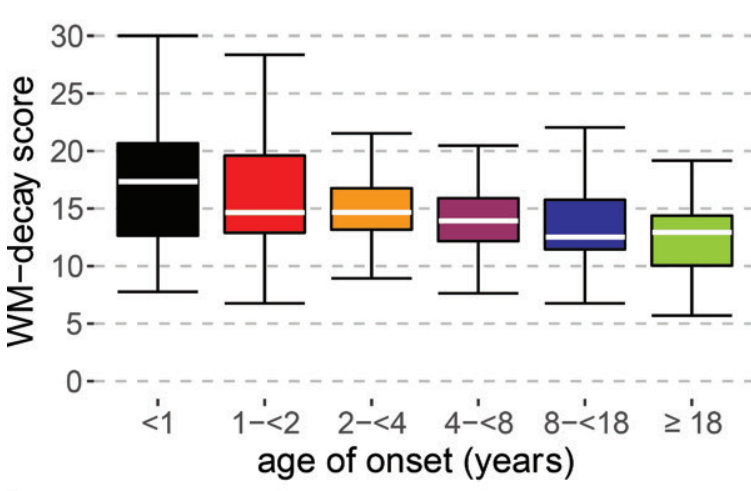

b.

score and rarefied or cystic white matter volume are lower (all $P<.001)$, and rate of progression is slower $(P<.001)$. With infantile onset, the VSR is also high.

MRI shows that in VWM, not only the rate of white matter decay but also pathologic type correlate with age at onset. With infantile onset, the white matter disappears within several months and may in part collapse, leading to ventricular dilatation. With onset in late infancy or early childhood, white matter may also
Figure 4: (a) Graphs show the white matter decay score per age-at-onset group, plotted over the disease duration on a log scale to prevent cluttered data points close to disease onset. Negative values indicate presymptomatic examinations. Thirty-two examinations were scored in group 1, 48 in group 2,81 in group 3, 68 in group 4, 48 in group 5, and 46 in group 6. Examinations of the same patients are connected. The slope of the change in white matter decay score is steeper for patients in the lower age-at-onset groups. (b) Box plots show VSR according to age of onset, with medians (white bar), first and third quartiles (box) and the minimum and maximum values within 1.5 times the interquartile range (whiskers). The median white matter decay score is higher in the lower age-at-onset groups $(P<.01)$ rapidly disappear, but it often has a swollen appearance, sometimes with progressive macrocephaly (6). MRI evidence of gliosis is lacking. With later childhood or adolescent onset, rarefaction and cystic white matter degeneration are more gradual and incomplete, and gliosis appears. With adult onset, white matter atrophy and gliosis are predominant, whereas rarefaction and cystic decay are mild or absent. These are general trends because of biologic variation without sharp borders between the age-at-onset groups. 


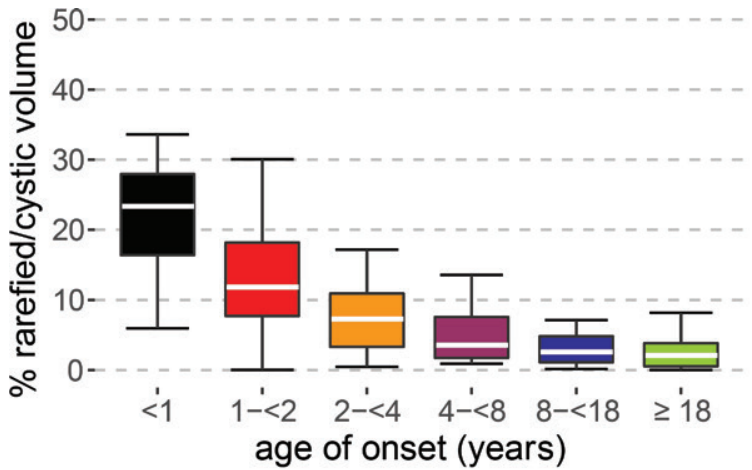

a.

Figure 5: Box plots of the 11 examinations segmented in group 1, 22 in group 2, 40 in group 3, 28 in group 4, 26 in group 5, and 22 in group 6 , with medians (white bar), first and third quartiles (box), and the minimum and maximum values within 1.5 times the interquartile range (whiskers) Volumes are represented as percentage of supratentorial intracranial volume. (a) The segmented rarefied or cystic white matter in percentage of supratentorial volume is higher in patients with a younger age at onset. (b) The segmented cerebrospinal fluid (CSF) in percentage of supratentorial volume is higher in patients with an older age at onset $(P<.01)$.

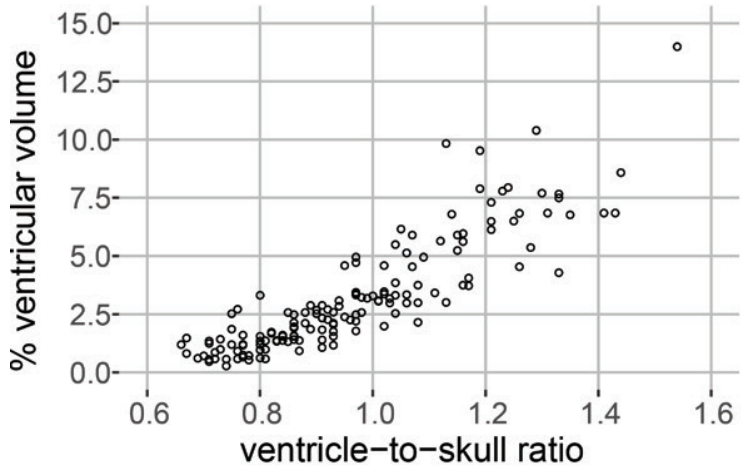

a.

Figure 6: Plots show comparison between methods. (a) Scatterplot with the segmented ventricular volume in percentage of supratentorial volume and the ventricle-to-skull ratio (VSR). The VSR is different from 0 , even when the segmented ventricular volume is close to $0 \%$. (b) Bland-Altman plot of the difference of the segmented versus the visually determined percentage of fluid-attenuated inversion recovery (FLAIR)-hyperintense volume. There is a mean difference \pm standard deviation of 1.3\% \pm 13.9 ; blue line (center) indicates mean difference, and red lines indicate the $95 \% \mathrm{Cl}$. When the FLAIR-hyperintense volume is low, the visual score is relatively higher, and vice versa.

Our findings are not entirely in line with a clinical natural history study (6) that suggested that age at onset correlates with rate of deterioration only for onset before age 4 years. In addition, the relapsing-remitting clinical course is not reflected in relapsingremitting cerebral white matter abnormalities; we did not observe improvement in any patient.

MRI findings align with VWM histopathologic findings, which are characterized by dysfunctional oligodendrocytes and astrocytes. Oligodendrocytes fail to produce and maintain sufficient myelin (3). Astrocyte dysfunction leads to meager or absent gliotic scar tissue (3). Astrocyte abnormality is primary and oligodendrocyte dysfunction is secondary (18). Disease severity is determined by the severity of astrocyte dysfunction, explaining the complete cystic white matter decay in early-onset cases and relatively adequate gliosis in late-onset cases. For comparison, Alexander disease is also an astrocytic disease (19) and, similar to VWM, is characterized by cystic white matter decay in severely affected patients with early onset, but not in patients with late onset (20). By contrast, in metachromatic leukodystrophy and Krabbe disease, oligodendrocyte and myelin abnormalities are primary; even in severely affected patients, no cystic white matter decay occurs $(21,22)$.

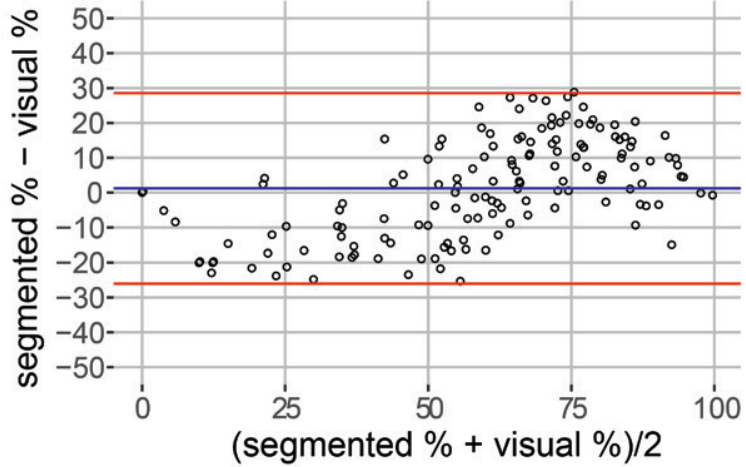

b.

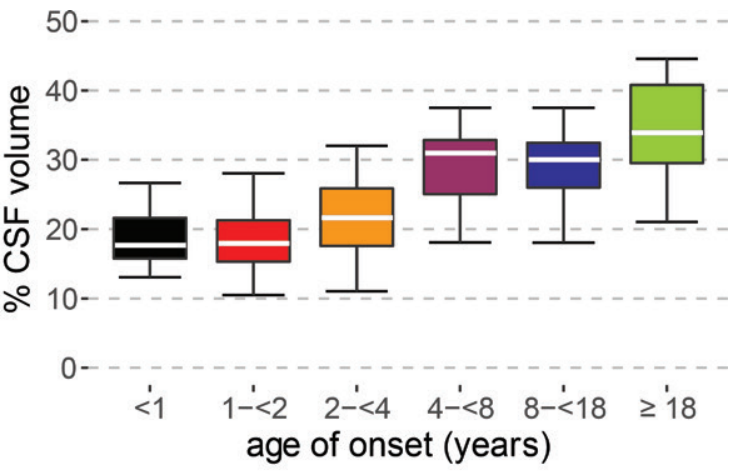

b. 
a similar appearance. In VWM, the inner rim of the corpus callosum is typically affected (2), whereas corpus callosum abnormalities are uncommon in vascular leukoencephalopathies. Basal ganglia abnormalities are typical in vascular leukoencephalopathies (28) but absent in VWM. Multiple sclerosis with extensive and cavitary lesions is another differential diagnosis. Isolated juxtacortical lesions and lesions perpendicular to the lateral ventricle are most discriminative for multiple sclerosis (25).

Our study had limitations. The degree of neurologic disability at the time of MRI examination was not recorded, preventing direct clinical-radiologic comparisons. The MRI examinations were collected over several decades, and many lack more advanced techniques, such as diffusion tensor imaging. Because of the retrospective and multicenter nature of the study, numerous different imaging protocols had been used; thus, a study of quantitative measures was not possible. To include all available MRI examinations, we applied three methods. For the digitized films, only the VSR could be used. For the segmented images, the VSR was shown to correlate well with the ventricular volume, thereby underlining its value. The white matter decay score was developed to include digital MRI examinations unsuitable for segmentation. Given the high intra- and interobserver reliability and strong correlations between the methods, the white matter decay score worked well but required training to calculate. Differences in section thickness led to a reduced quality of segmentation, despite interpolation to isometric voxels. In prospective studies, isotropic (T1-weighted and FLAIR) three-dimensional sequences with predefined parameters would be desirable.

Quantitative techniques are superior to conventional techniques to assess microstructural tissue integrity, such as myelin content (29), axonal integrity (30), and g-ratio (31). These methods have not been evaluated in VWM. Although promising, results should be interpreted with caution. In VWM, axons and myelin sheaths may be thin, and myelin may be uncompacted (32) and changed in composition (33), thereby altering T1, T2, and magnetization transfer. Estimations of the microstructural parameters are based on normal white matter and may therefore be incorrect in VWM. Future studies will benefit from including quantitative techniques.

In conclusion, the pathologic type and time course of cerebral white matter decay in vanishing white matter depend on age at onset. The earlier the onset, the more cystic and rapid the white matter decay, whereas with a late onset, white matter atrophy and gliosis predominate. Insight into these MRI features is essential for proper interpretation of MRI findings. It is crucial to consider the natural MRI history in upcoming therapeutic trials.

Acknowledgments: The authors thank all referring physicians and IT staff for technical support in digitizing radiologic films.

Author contributions: Guarantors of integrity of entire study, M.D.S. M.S.v.d.K.; study concepts/study design or data acquisition or data analysis/interpretation, all authors; manuscript drafting or manuscript revision for important intellectual content, all authors; approval of final version of submitted manuscript, all authors; agrees to ensure any questions related to the work are appropriately resolved, all authors; literature research, M.D.S., M.S.v.d.K.; clinical studies, M.D.S., M.L.A., F.B., P.J.W.P., M.S.v.d.K.; statistical analysis, M.D.S., T.v.d.B., M.S.v.d.K.; and manuscript editing, M.D.S., M.L.A., F.B., P.J.W.P., M.S.v.d.K.

Disclosures of Conflicts of Interest: M.D.S. disclosed no relevant relationships M.L.A. disclosed no relevant relationships. Tv.d.B. disclosed no relevant rela- tionships. F.B. disclosed no relevant relationships. P.J.W.P. disclosed no relevant relationships. M.S.v.d.K. disclosed no relevant relationships.

\section{References}

1. van der Knaap MS, Pronk JC, Scheper GC. Vanishing white matter disease. Lancet Neurol 2006;5(5):413-423.

2. van der Lei HD, Steenweg ME, Barkhof F, et al. Characteristics of early MRI in children and adolescents with vanishing white matter. Neuropediatrics 2012;43(1):22-26

3. Bugiani M, Vuong C, Breur M, van der Knaap MS. Vanishing white matter: a leukodystrophy due to astrocytic dysfunction. Brain Pathol 2018;28(3):408-421.

4. Leegwater PA, Vermeulen G, Könst AA, et al. Subunits of the translation initiation factor eIF2B are mutant in leukoencephalopathy with vanishing white matter. Nat Genet 2001;29(4):383-388.

5. van der Knaap MS, Leegwater PA, Könst AA, et al. Mutations in each of the five subunits of translation initiation factor eIF2B can cause leukoencephalopathy with vanishing white matter. Ann Neurol 2002;51(2):264-270.

6. Hamilton EMC, van der Lei HDW, Vermeulen G, et al. Natural history of vanishing white matter. Ann Neurol 2018;84(2):274-288.

7. Dooves S, Bugiani M, Wisse LE, Abbink TEM, van der Knaap MS, Heine VM. Bergmann glia translocation: a new disease marker for vanishing white matter identifies therapeutic effects of Guanabenz treatment. Neuropathol Appl Neurobiol 2018;44(4):391-403.

8. Abbink TEM, Wisse LE, Jaku E, et al. Vanishing white matter: deregulated integrated stress response as therapy target. Ann Clin Transl Neurol 2019;6(8):1407-1422.

9. Wong YL, LeBon L, Edalji R, Lim HB, Sun C, Sidrauski C. The small molecule ISRIB rescues the stability and activity of vanishing white matter disease eIF2B mutant complexes. eLife 2018;7:e32733.

10. Fogli A, Schiffmann R, Bertini E, et al. The effect of genotype on the natural history of eIF2B-related leukodystrophies. Neurology 2004;62(9):1509-1517.

11. Slynko I, Nguyen S, Hamilton EMC, et al. Vanishing white matter: eukaryotic initiation factor $2 \mathrm{~B}$ model and the impact of missense mutations. Mol Genet Genomic Med 2021. 10.1002/mgg3.1593. Published online January 12, 2021

12. van der Knaap MS, Barth PG, Gabreëls FJ, et al. A new leukoencephalopathy with vanishing white matter. Neurology 1997;48(4):845-855.

13. van der Knaap MS, Kamphorst W, Barth PG, Kraaijeveld CL, Gut E, Valk J. Phenotypic variation in leukoencephalopathy with vanishing white matter. Neurology 1998;51(2):540-547.

14. van der Knaap MS, van Berkel CG, Herms J, et al. eIF2B-related disorders: antenatal onset and involvement of multiple organs. Am J Hum Genet 2003;73(5):1199-1207.

15. van der Knaap MS, Bakker CJ, Faber JA, et al. Comparison of skull circumference and linear measurements with CSF volume MR measurements in hydrocephalus. J Comput Assist Tomogr 1992;16(5):737-743.

16. Valverde S, Salem M, Cabezas M, et al. One-shot domain adaptation in multiple sclerosis lesion segmentation using convolutional neural networks. Neuroimage Clin 2019;21:101638.

17. Fleiss JL. The design and analysis of clinical experiments. New York, NY: Wiley, 1986; 149-185.

18. Dooves S, Bugiani M, Postma NL, et al. Astrocytes are central in the pathomechanisms of vanishing white matter. J Clin Invest 2016;126(4):1512-1524.

19. Brenner M, Johnson AB, Boespflug-Tanguy O, Rodriguez D, Goldman JE, Messing A. Mutations in GFAP, encoding glial fibrillary acidic protein, are associated with Alexander disease. Nat Genet 2001;27(1):117-120.

20. Dinopoulos A, Gorospe JR, Egelhoff JC, et al. Discrepancy between neuroimaging findings and clinical phenotype in Alexander disease. AJNR Am J Neuroradiol 2006;27(10):2088-2092.

21. Krieg SI, Krägeloh-Mann I, Groeschel S, et al. Natural history of Krabbe disease - a nationwide study in Germany using clinical and MRI data. Orphanet J Rare Dis 2020;15(1):243.

22. Groeschel S, Kehrer C, Engel C, et al. Metachromatic leukodystrophy: natural course of cerebral MRI changes in relation to clinical course. J Inherit Metab Dis 2011;34(5):1095-1102.

23. Invernizzi F, Tigano M, Dallabona $\mathrm{C}$, et al. A homozygous mutation in LYRM7/MZM1L associated with early onset encephalopathy, lactic acidosis, and severe reduction of mitochondrial complex III activity. Hum Mutat 2013;34(12):1619-1622.

24. Melchionda L, Haack TB, Hardy S, et al. Mutations in APOPT1, encoding a mitochondrial protein, cause cavitating leukoencephalopathy with cytochrome c oxidase deficiency. Am J Hum Genet 2014;95(3):315-325. 
25. Ayrignac X, Menjot de Champfleur N, Menjot de Champfleur S, et al. Brain magnetic resonance imaging helps to differentiate atypical multiple sclerosis with cavitary lesions and vanishing white matter disease. Eur J Neurol 2016;23(6):995-1000.

26. van der Lei HD, Steenweg ME, Bugiani M, et al. Restricted diffusion in vanishing white matter. Arch Neurol 2012;69(6):723-727.

27. van der Voorn JP, Pouwels PJ, Kamphorst W, et al. Histopathologic correlates of radial stripes on MR images in lysosomal storage disorders. AJNR Am J Neuroradiol 2005;26(3):442-446.

28. Tikka S, Baumann M, Siitonen M, et al. CADASIL and CARASIL. Brain Pathol 2014;24(5):525-544.

29. Piredda GF, Hilbert T, Thiran JP, Kober T. Probing myelin content of the human brain with MRI: a review. Magn Reson Med 2021;85(2):627-652.
30. Zhang H, Schneider T, Wheeler-Kingshott CA, Alexander DC. NODDI: practical in vivo neurite orientation dispersion and density imaging of the human brain. Neuroimage 2012;61(4):1000-1016.

31. Jung W, Lee J, Shin HG, et al. Whole brain g-ratio mapping using myelin water imaging (MWI) and neurite orientation dispersion and density imaging (NODDI). Neuroimage 2018;182:379-388.

32. Klok MD, Bugiani M, de Vries SI, et al. Axonal abnormalities in vanishing white matter. Ann Clin Transl Neurol 2018;5(4):429-444.

33. Blüml S, Philippart M, Schiffmann R, Seymour K, Ross BD. Membrane phospholipids and high-energy metabolites in childhood ataxia with CNS hypomyelination. Neurology 2003;61(5):648-654. 